ORIGINAL PAPER

\title{
The POTENTIAL PROgNOSTIC ROLE OF PERITUMORAL \\ EOSINOPHILS WITHIN WHOLE TUMOR-ASSOCIATED \\ INFLAMMATORY CELLS AND STROMAL HISTOLOGICAL \\ CHARACTERISTICS IN COLORECTAL CANCER
}

Saime Ramadan ${ }^{1}$, Burcu Saka ${ }^{2}$, Enver Yarikkaya ${ }^{3}$, Ahmet Bilici $^{4}$, Mustafa Oncel $^{5}$

\begin{abstract}
${ }^{1}$ Department of Pathology, Baskent University Istanbul Hospital, Istanbul, Turkey
${ }^{2}$ Department of Pathology, Istanbul Medipol University Hospital, Istanbul, Turkey

${ }^{3}$ Department of Pathology, Istanbul Training and Research Hospital, Istanbul, Turkey

${ }^{4}$ Department of Medical Oncology, Istanbul Medipol University Hospital, Istanbul, Turkey

${ }^{5}$ Department of General Surgery, Istanbul Medipol University Hospital, Istanbul, Turkey
\end{abstract}

\begin{abstract}
We aimed to determine the prognostic role of whole tumor-associated inflammatory cells, especially eosinophils, and stromal histological characteristics in relation to other prognostic parameters in patients with colorectal carcinoma (CRC). A total of 122 patients who underwent an operation for CRC were included in this retrospective study. Conventional (tumor grade, TNM stage and venous invasion [VI]) and other histopathological (intratumoral/peritumoral budding [ITB/PTB], desmoplasia) tumor parameters were recorded and classified by density, as were the tumor-associated inflammatory parameters (intratumoral/peritumoral lymphocytes [ITL/PTL], eosinophils [IE/PTE], overall inflammation [ITI/PTI], Crohn-like inflammation [CLI]). Cancer-specific survival data were analyzed with respect to all tumor parameters. High ITB and PTB were significantly correlated with a higher rate of pT4, VI and desmoplasia $(\mathrm{p}<0.05)$. An association of moderate ITL and extensive PTL with lesser likelihood of VI and metastasis; an association of extensive CLI with a significantly lower rate of metastasis and TNM stage IV; and minimal PTE with a significantly higher rate of pT4 stage, metastasis and ITB were detected ( $\mathrm{p}<0.05$ for each). Our findings revealed that low score tumoral budding and an increase in tumor-related inflammation were associated with lesser likelihood of poor prognostic tumor parameters. Nonetheless, given the association of an increase in PTE with lesser likelihood of ITB, pT4, metastasis, and with non-significantly for better survival rates, our findings emphasize the potential role of peritumoral eosinophils as an additional prognostic parameter in CRC.
\end{abstract}

Key words: colorectal cancer, survival, tumor-related inflammation, tumor budding.

\section{Introduction}

Colorectal cancer (CRC) is one of the most common cancers worldwide and the third leading cause of cancer-related mortality, despite better understanding of its pathogenesis and advances in therapy. Colorectal cancer incidence has been increasing steadily, especially in developing countries. Obesity, sedentary life style, red meat consumption, alcohol and tobacco are the major risk factors behind the growth of CRC [1]. 
Colon cancer and rectal cancer have similar histopathologic risk factors and similar survival rates $[1,2]$.

Colorectal cancer comprises a group of diseases driven by several mutations and mutagens. Hereditary CRCs account for 7-10\% of all cases and include Lynch syndrome, adenomatous and hamartomatous syndromes [3]. Up to $30 \%$ of CRC patients have a family history of the neoplasm, meaning there are probably predisposing germ-line mutations. To date, several nuclear DNA variants have been shown to be associated with increased risk of CRC. However, there are conflicting results about the role of inherited mitochondrial DNA (mtDNA) mutations in CRC carcinogenesis. Previous studies suggested that certain polymorphic mtDNA positions were associated with increased risk of CRC in different nations $[4,5]$ but a British study [6] and a recent Polish [7] study did not support the hypothesis that mtDNA variants contribute to inherited predisposition of CRC.

Currently, post-surgical staging according to the American Joint Committee on Cancer (AJCC)/Union for International Cancer Control (UICC), tumor node metastasis (TNM) system is a standard reference for prognosis and a guide for therapeutic decision making in CRC [8]. However, TNM classification reflects an anatomical extent rather than biological tumor characteristics [9]. Alongside considerable variation in the clinical course of disease among patients with an identical stage $[8,10]$, unexpected progression during the follow-up of early stage, lymph node negative patients causes difficulties to decide the therapeutic modalities [9, 10, 11, 12]. Tumor budding seems important in this regard, as a strong adverse prognostic factor to select patients for adjuvant therapy particularly in those with early stage, lymph node negative disease [12, 13].

However, given the heterogeneity of tumor biology within each stage, there is a need for additional stage-independent prognostic histopathological parameters to identify patients with more aggressive clinical behavior. This will guide clinical decisions in terms of follow-up scheduling and provision of adjuvant therapy tailored to individualized risk of progression $[8,10,12]$.

In accordance with the increasingly apparent recognition of CRC outcome to be dependent not only on tumor characteristics but also on the interactions between host immune/inflammatory response and tumor [14], assessment of tumor-related local inflammation has been proposed as a novel prognostic parameter [10, 14].

Several studies to date have confirmed the prognostic value of the tumor-related inflammatory or immune cell infiltrate especially the adaptive immune response (i.e. infiltration of $\mathrm{T}$ cells) in patients with CRC [14, 15, 16]. For example, Galon et al. found a positive correlation between the presence of mark- ers for Th1 polarization of cytotoxic and memory $\mathrm{T}$ cells and a low incidence of tumor recurrence [15]. An international concortitum suggested the Immunoscore assay to assess the prognostic value of total tumor-infiltrating T-cell counts and cytotoxic tumor-infiltrating T-cell counts in patients with stage I-III colon cancer. They found that the Immunoscore provides a reliable estimate of the risk of recurrence in patients with colon cancer [16]. There are a few studies that have focused on the innate immune cells (i.e. eosinophils) with a standardized assessment regarding type, density and location of inflammatory infiltrate $[17,18,19]$. A simplified histopathology-based scoring method described by Klintrup et al. [18] is important in this regard, since it represents standard criteria for the prognostic value of tumorrelated inflammatory infiltrate assessments.

This study was therefore designed to investigate the prognostic role of tumor-associated inflammatory parameters and tumor budding in relation to other prognostic tumor parameters in patients operated on CRC. Tumor-associated inflammatory infiltrates were assessed based on standardized criteria being stratified by location (intratumoral, peritumoral), inflammatory cell subtype (lymphocyte, eosinophil, overall inflammation) and density.

\section{Material and methods}

\section{Study population}

A total of 122 patients operated on for colorectal cancer (mean (SD) age: 59.9 (12.4) years, 59.0\% were men) were included in this retrospective study that was conducted at a tertiary care center between 2013 and 2014 (Table I). Rare histologic subtypes such as medullary and squamous cell carcinoma were excluded from the study and patients with insufficient disease information were excluded from data analysis.

The study was conducted in full accordance with local good clinical practice guidelines and current legislations, while permission was obtained from our institutional ethics committee for the use of patient data for publication purposes. The need for consent from the patients was waived because of the retrospective nature of this study.

\section{Study parameters}

Data on patient demographics (age, gender), tumor grade and $\mathrm{pT}$ and $\mathrm{pN}$ stages, and neoadjuvant therapy history were retrospectively obtained from patient charts. Metastases were recorded both at the time of diagnosis and during follow-up. Intratumoral (ITB), peritumoral budding (PTB), venous invasion and desmoplasia were evaluated in addition to tumorassociated inflammatory parameters (intratumoral 
lymphocyte [ITL], peritumoral lymphocyte [PTL], Crohn-like inflammation [CLI], intratumoral eosinophils [IE], peritumoral eosinophils [PTE], intratumoral overall inflammation [ITI], peritumoral overall inflammation [PTI]). Survival data were obtained from patients' medical records or via phone call interviews.

The prognostic role of tumor-associated inflammatory parameters and desmoplasia in relation to conventional prognostic parameters were evaluated, along with 5-year cancer-specific survival (CSS) rate and mean CSS time. The relation of tumor budding with conventional prognostic parameters, tumor-related inflammatory parameters and desmoplasia was also analyzed along with the concordance between ITB and PTB.

\section{Histopathology}

For the classification of TNM, AJCC/UICC 2017 criteria were used [20], while histological tumor type and differentiation (grade) were assessed based on the 2010 edition of the World Health Organization classification [21].

We analyzed venous invasion on hematoxylin and eosin (HE) slides with attention to the orphan arteriole and protruding tongue signs. An orphan arteriole sign is described as a circumscribed tumor nodule adjacent to a muscularized artery without an obvious accompanying vein. The protruding tongue sign is described as a smooth bordered protrusion of a tumor into pericolic fat adjacent to an artery [22]. Besides these morphologic findings, many studies have demonstrated the superiority of elastin staining compared to (HE) alone in the detection of VI $[22,23]$. Since determination of the true prevalence of VI is difficult based on $\mathrm{HE}$, especially when the muscular wall of the vein is obliterated, elastin stain was also performed on all tumor blocks. Venous invasion was considered positive when a tumor was observed in an endothelium-lined space [22].

Both intratumoral and peritumoral budding were assessed on HE slides as described previously by Gra-
Table I. Demographic and clinical characteristics of the study group

\begin{tabular}{|c|c|c|}
\hline \multirow{2}{*}{$\begin{array}{l}\text { AGE, YEARS } \\
\text { (MEAN, RANGE) }\end{array}$} & 60 & $28-87$ \\
\hline & $\mathrm{N}$ & $\%$ \\
\hline \multicolumn{3}{|l|}{ Gender } \\
\hline Male & 72 & 59 \\
\hline Female & 50 & 41 \\
\hline \multicolumn{3}{|l|}{ Grade } \\
\hline Low & 107 & 87.7 \\
\hline High & 15 & 12.3 \\
\hline \multicolumn{3}{|l|}{$\mathrm{T}$} \\
\hline $1-2$ & 23 & 18.8 \\
\hline 3 & 70 & 53.4 \\
\hline 4 & 29 & 27.8 \\
\hline \multicolumn{3}{|l|}{$\mathrm{N}$} \\
\hline 0 & 72 & 59.0 \\
\hline 1 & 33 & 26.9 \\
\hline 2 & 17 & 14.1 \\
\hline \multicolumn{3}{|l|}{ Metastasis } \\
\hline No & 97 & 79.5 \\
\hline Yes & 25 & 20.5 \\
\hline \multicolumn{3}{|l|}{ TNM Stage } \\
\hline I & 21 & 17.2 \\
\hline II & 44 & 36.1 \\
\hline III & 35 & 28.7 \\
\hline IV & 22 & 18.0 \\
\hline
\end{tabular}

ham et al. [24]. The intensity of budding was scored accordingly as no budding, low (1-9 budding foci), and high ( $\geq 10$ foci) (Figs. 1, 2). Desmoplasia was scored semi-quantitatively as minimal, moderate and severe.
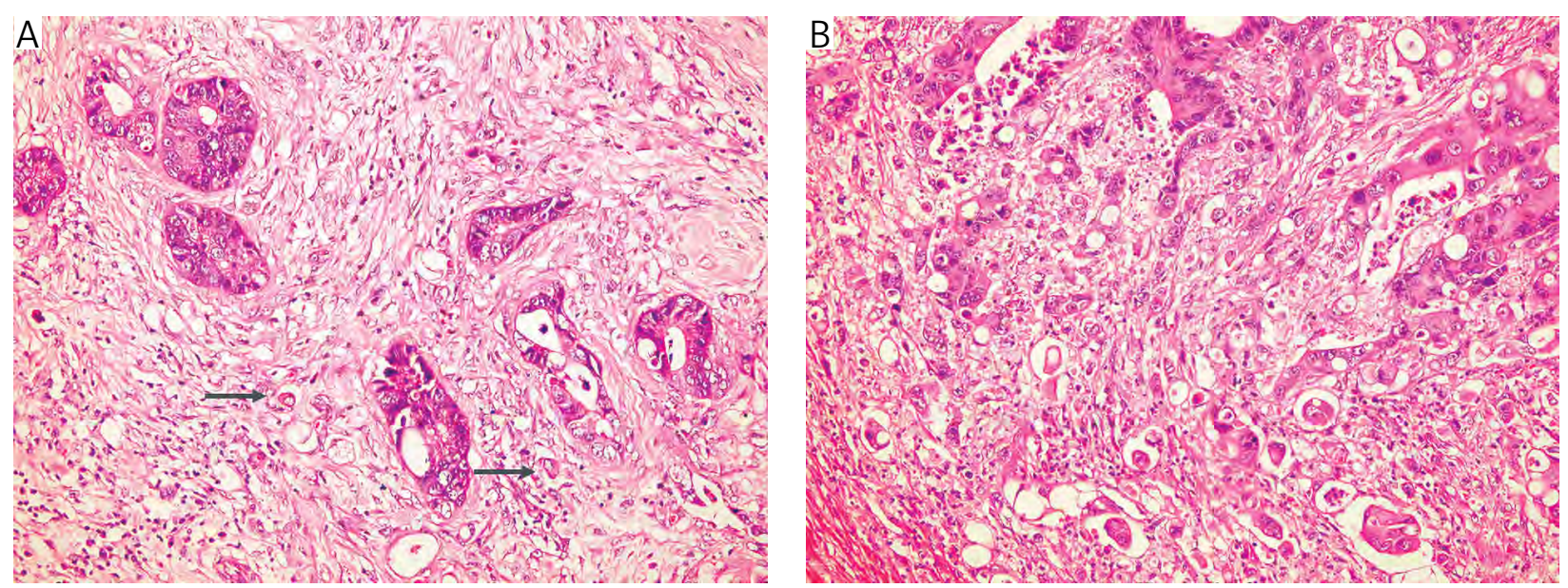

Fig. 1. Low (shown by arrows) and high score intratumoral budding (HE, magnification $200 \times$ ) 

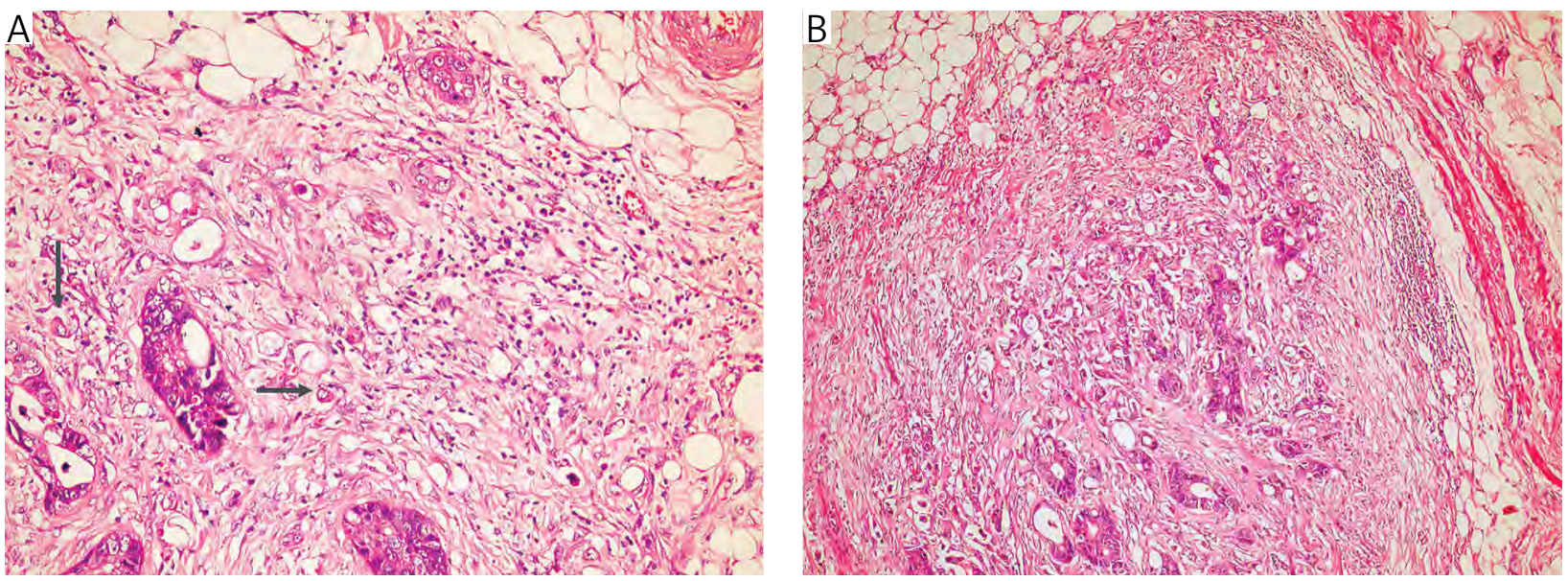

Fig. 2. Low (shown by arrows) and high score peritumoral budding (HE, magnification $200 \times$ )
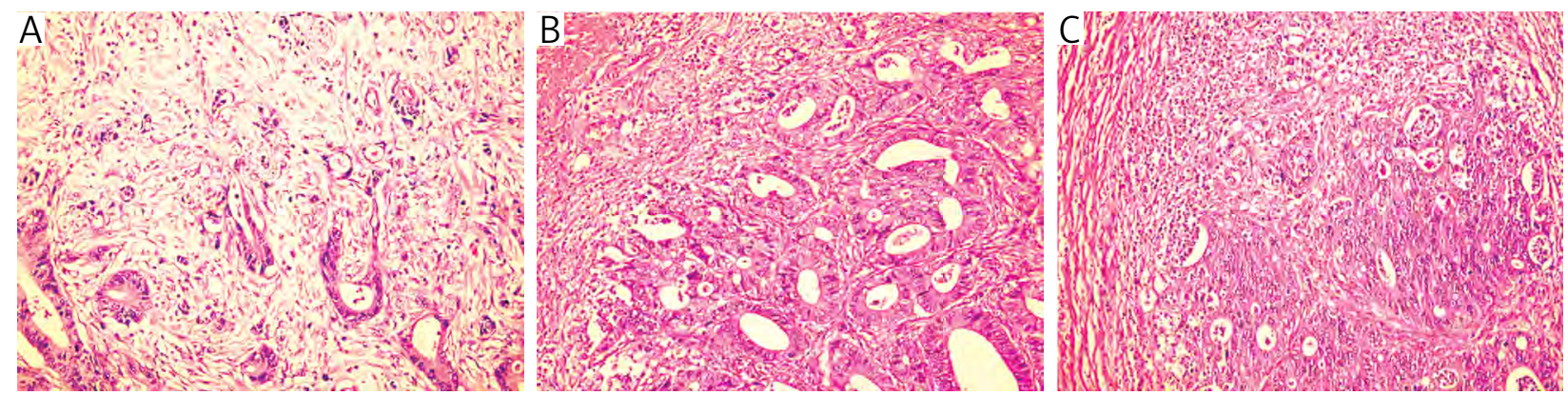

Fig. 3. The intensity of overall inflammatory cell reaction in order to minimal, moderate and severe (HE, magnification $200 \times$ )
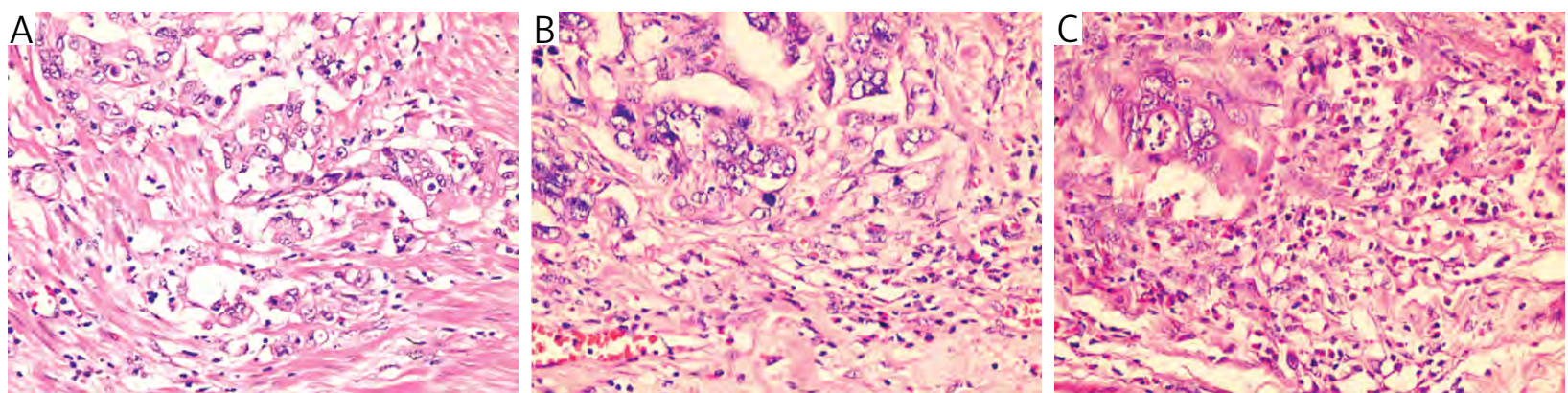

Fig. 4. The intensity of eosinophil infiltration in order to minimal, moderate and severe (HE, magnification $400 \times$ )

\section{Tumor-associated inflammation}

The intensity of overall inflammatory cell reaction was assessed separately within the tumor center (intratumoral) and in the stroma at the invasive tumor margin (peritumoral) with a modified method described by Klintrup et al. [18] and scored from 1 to 3 based on a mild or patchy increase (score 1), a prominent inflammatory reaction (score 2), and a florid "cuplike" inflammatory infiltrate (score 3; Fig. 3).

The numbers of intratumoral and peritumoral eosinophils were evaluated separately on HE stained slides, using a $40 \times$ objective lens in a high power field (HPF) measuring $0,24 \mathrm{~mm}^{2}$ (Olympus BX45).
IE and PTE were categorized according to modified Fernandez-Acenero criteria, considering of $<10$ cells/ $0.24 \mathrm{~mm}^{2}$ area as minimal, $10-50$ cells $/ 0.24 \mathrm{~mm}^{2}$ area as moderate and $>50$ cells $/ 0.24 \mathrm{~mm}^{2}$ area as extensive infiltration [25] (Fig. 4).

Crohn-like response was scored as none or mild-tomoderate and marked according to College of American Pathologists (CAP) criteria [26]. Intratumoral lymphocytes were evaluated based on CAP criteria and graded as none, mild-to-moderate (1-2 per HPF) and marked ( $\geq 3$ per HPF), (Fig. 5).

Peritumoral lymphocytes were evaluated according to modified Huh et al. [27] criteria on HE stained slides and categorized as minimal, moderate and severe (Fig. 6). 

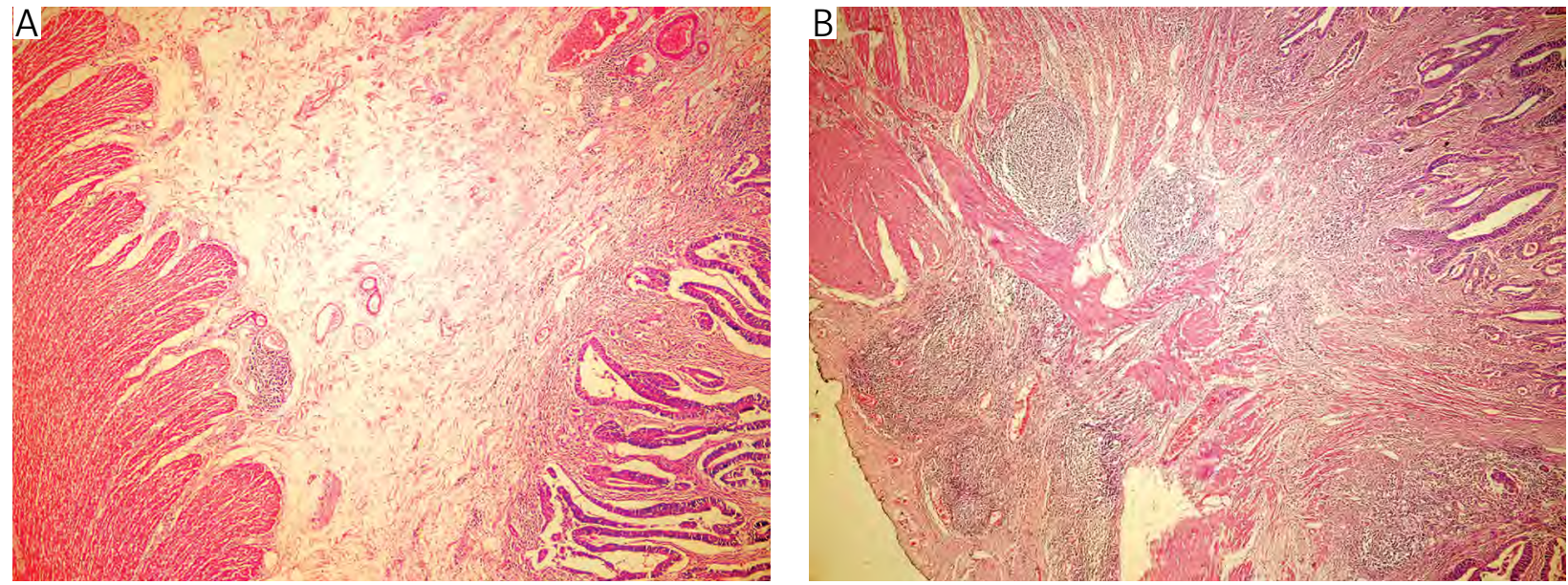

Fig. 5. Crohn-like response: minimal and marked (HE, magnification $40 \times$ )
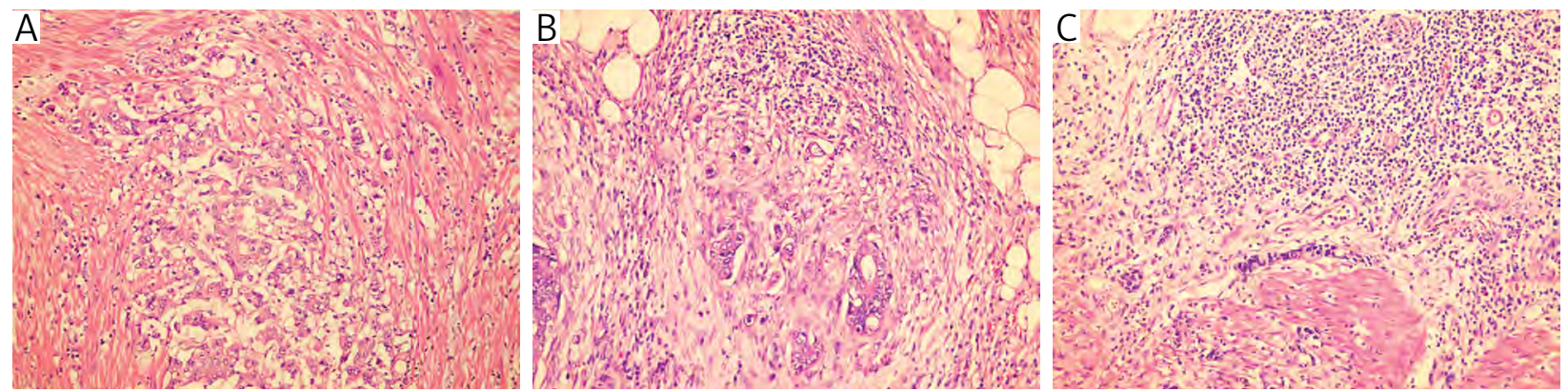

Fig. 6. Peritumoral lymphocyte infiltration in order to minimal, moderate and severe (HE, magnification $200 \times$ )

\section{Statistical analysis}

Statistical analysis was performed using IBM SPSS Statistics for Windows, version 22.0 (IBM Corp., Armonk, NY). The $\chi^{2}$ test was used for the comparison of categorical data, while the one way ANOVA test was used for the parametric variables. The $\kappa$ concordance index was used to determine the concordance between intratumoral and peritumoral budding. Cancer-specific survival was assessed using the $\mathrm{Ka}$ plan-Meier method and the significance among patient groups was calculated using the log-rank test. Cox proportional hazards regression model was used to determine hazard ratios and data were expressed as "mean (standard deviation; SD)", minimum-maximum, 95\% confidence interval (CI) and percent (\%) where appropriate. $\mathrm{P}<0.05$ was considered statistically significant.

\section{Results}

Tumor-associated inflammatory parameters and tumor budding according to grade, TNM stage and venous invasion

Tumor-associated inflammatory parameters and tumor budding according to TNM stage are shown in Table II.
High ITB as compared with the absence of ITB and low ITB was associated with significantly higher rate of pT4 (42.0\% vs. $6.3 \%$ and $12.5 \%$, respectively, $\mathrm{p}=0.002)$ and venous invasion $(54.0 \%$ vs. $18.7 \%$ and $33.9 \%$, respectively, $\mathrm{p}=0.019$ ).

High PTB as compared with the absence of PTB and low PTB was associated with significantly higher rate of pT4 ( $41.5 \%$ vs. $6.3 \%$ and $11.3 \%$, respectively, p $=0.001)$ and TNM stage IV (28.3\% vs. $12.5 \%$ and $9.4 \%$, respectively, $\mathrm{p}=0.043$ ) alongside higher likelihood of venous invasion $(52.8 \%$ vs. $18.8 \%$ and $34.0 \%$, respectively, $\mathrm{p}=0.024$ ).

Most of our cases were low grade $(87.7 \%)$. We only found a statistically significant positive correlation with grade and ITL. Extensive ITL was strongly associated with a higher likelihood of high grade tumor rather than mild-to-moderate ITL $(26.9 \%$ vs. $7.2 \%, \mathrm{p}=0.033$ ). However extensive ITL was inversely correlated with venous invasion $(\mathrm{p}=0.048)$.

The presence of metastasis was more likely to occur in cases of minimal $(28.1 \%)$ and moderate (17.9\%) PTL in comparison to extensive (0.0\%) PTL $(\mathrm{p}=0.025)$.

Marked CLI as compared to the absence of CLI and mild-to-moderate CLI was associated with a significantly lower rate of metastasis and TNM stage IV $(p=0.005)$. 


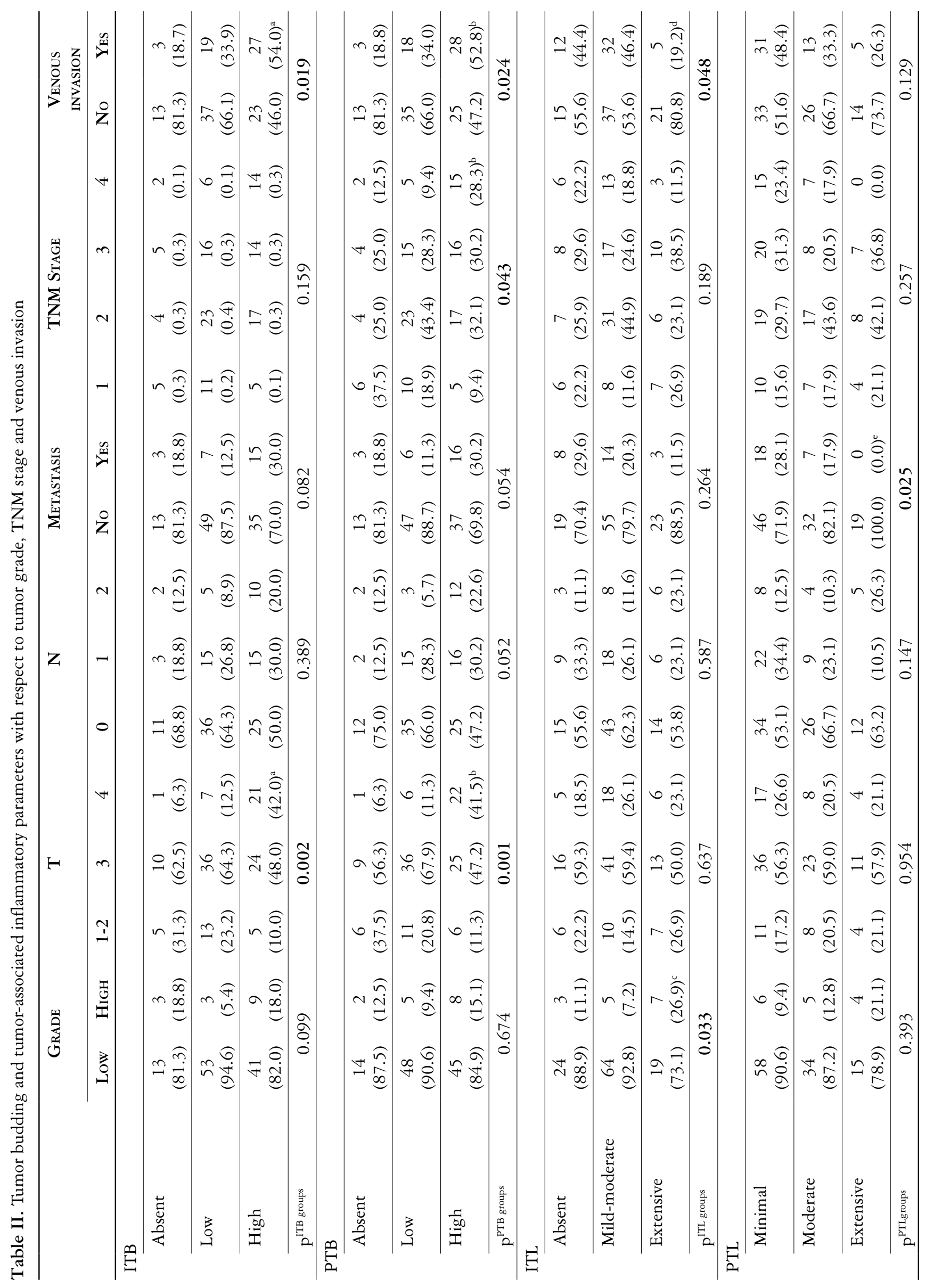




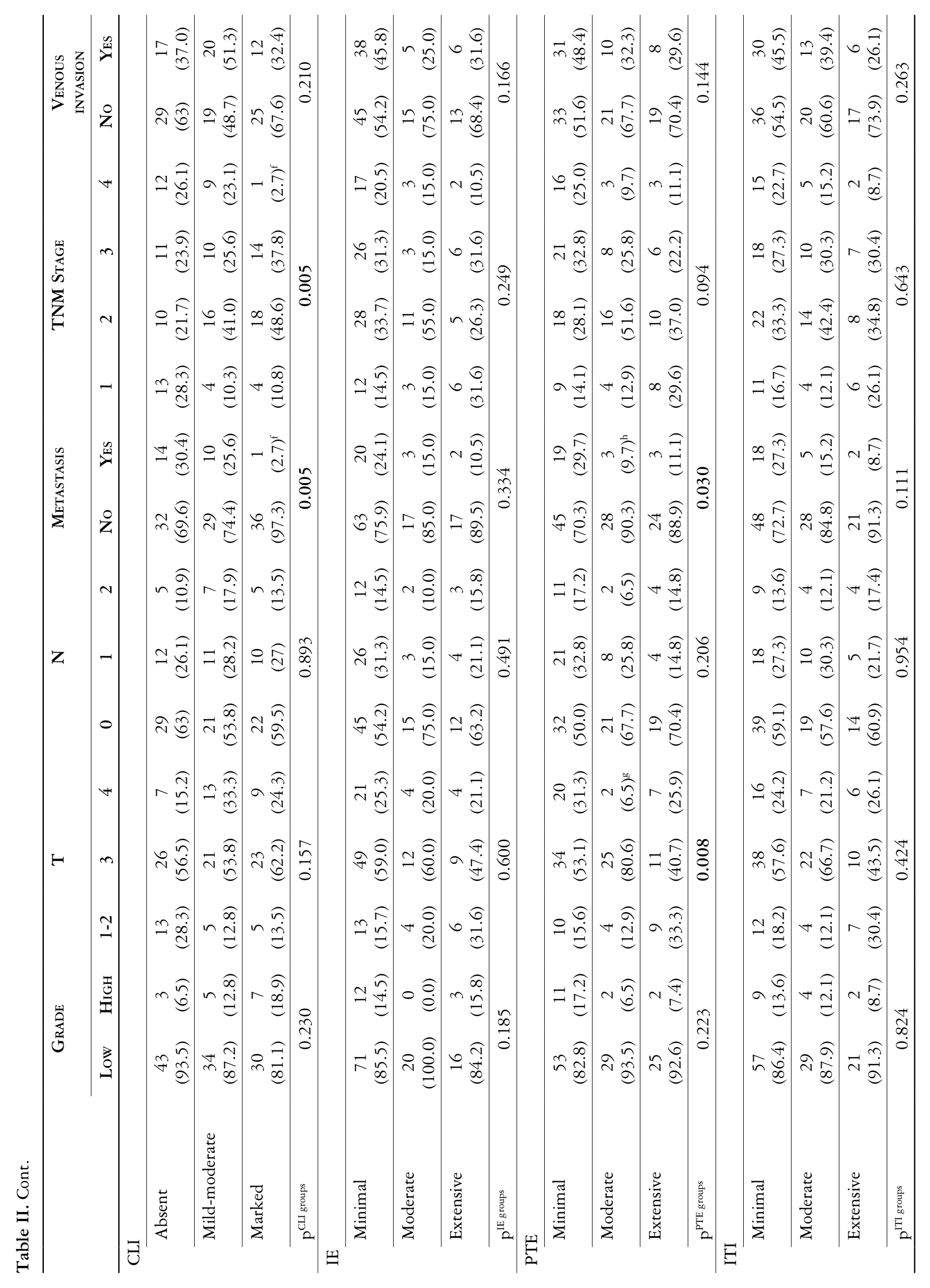




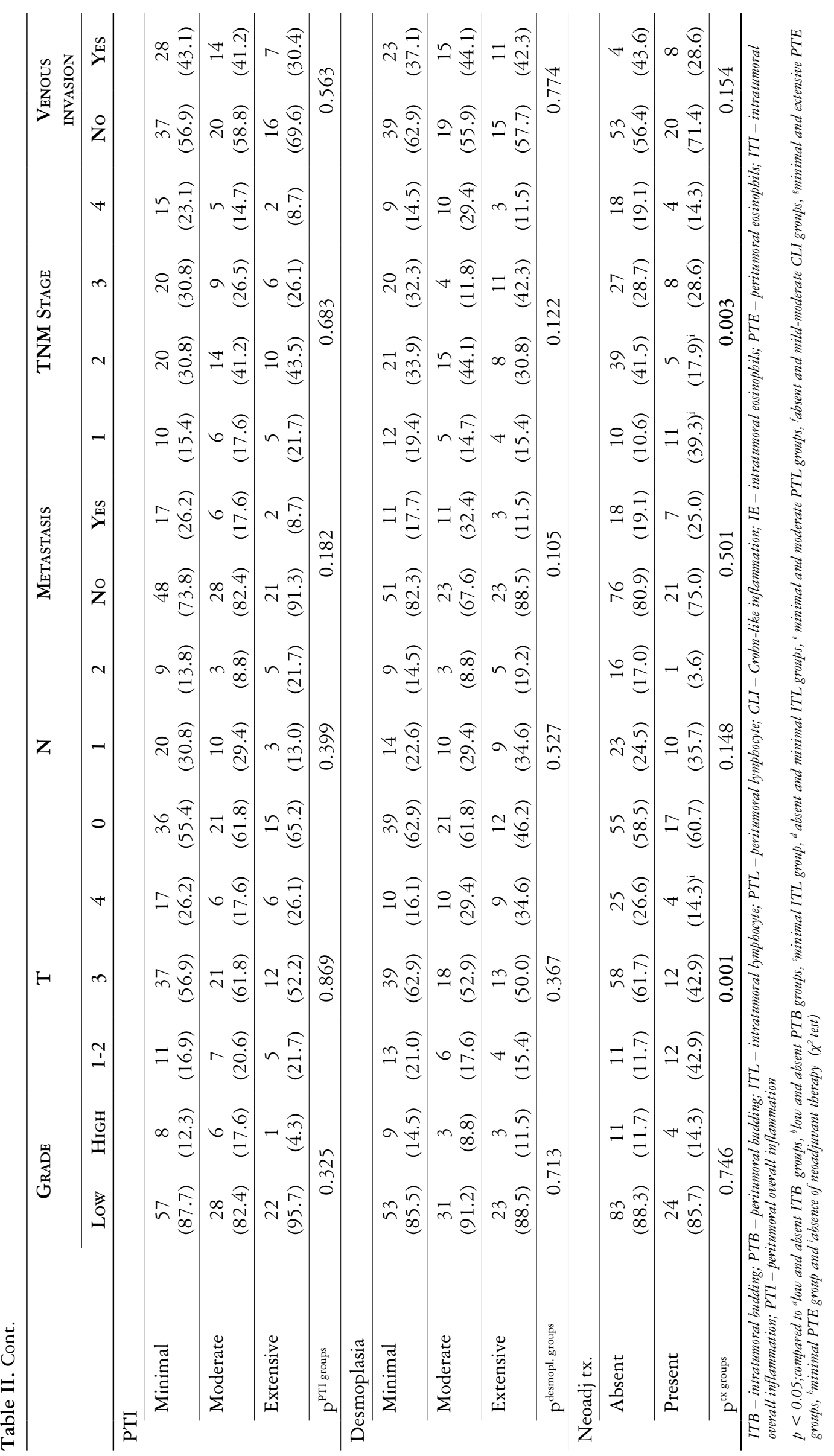


Table III. Concordance between ITB and PTB and association of tumor budding with study parameters

\begin{tabular}{|c|c|c|c|c|c|c|c|c|}
\hline & \multicolumn{4}{|c|}{ PTB } & \multicolumn{4}{|c|}{ ITB } \\
\hline & ABSENT & Low & HiGH & P VALUE & Absent & Low & HigH & P VALUE \\
\hline Age, mean (SD) & $57.8(15.5)$ & $59.5(12.7)$ & $61.0(11.3)$ & $0.628^{1}$ & $58.3(15.0)$ & $59.6(13.0)$ & $60.7(11.0)$ & $0.774^{1}$ \\
\hline \multicolumn{9}{|l|}{ Gender, n (\%) } \\
\hline Male & $9(56.3)$ & $34(64.2)$ & $29(54.7)$ & $0.596^{2}$ & $10(62.5)$ & $33(58.9)$ & $29(58.0)$ & $0.950^{2}$ \\
\hline Female & $7(43.8)$ & $19(35.8)$ & $24(45.3)$ & & $6(37.5)$ & $23(41.1)$ & $21(42.0)$ & \\
\hline \multicolumn{9}{|l|}{ ITL, n (\%) } \\
\hline Absent & $4(25.0)$ & $10(18.9)$ & $13(24.5)$ & $0.838^{2}$ & $4(25.0)$ & $11(19.6)$ & $12(24.0)$ & $0.767^{2}$ \\
\hline Mild-moderate & $8(50.0)$ & $30(56.6)$ & $31(58.5)$ & & $7(43.8)$ & $33(58.9)$ & $29(58.0)$ & \\
\hline Extensive & $4(25.0)$ & $13(24.5)$ & $9(17.0)$ & & $5(31.2)$ & $12(21.4)$ & $9(18.0)$ & \\
\hline \multicolumn{9}{|l|}{ PTL, n (\%) } \\
\hline Minimal & $10(62.5)$ & $24(45.3)$ & $30(56.6)$ & $0.283^{2}$ & $9(56.3)$ & $27(48.2)$ & $28(56.0)$ & $0.590^{2}$ \\
\hline Moderate & $2(12.5)$ & $20(37.7)$ & $17(32.1)$ & & $3(18.8)$ & $20(35.7)$ & $16(32.0)$ & \\
\hline Extensive & $4(25.0)$ & $9(17.0)$ & $6(11.3)$ & & $4(25.0)$ & $9(16.1)$ & $6(12.0)$ & \\
\hline \multicolumn{9}{|l|}{ CLI, n (\%) } \\
\hline Absent & $8(50.0)$ & $18(34.0)$ & $20(37.7)$ & $0.487^{2}$ & $7(43.8)$ & $21(37.5)$ & $18(36.0)$ & $0.821^{2}$ \\
\hline Mild-moderate & $3(18.8)$ & $16(30.2)$ & $20(37.7)$ & & $3(18.8)$ & $19(33.9)$ & $17(34.0)$ & \\
\hline Marked & $5(31.3)$ & $19(35.8)$ & $13(24.5)$ & & $6(37.5)$ & $16(28.6)$ & $15(30.0)$ & \\
\hline \multicolumn{9}{|l|}{ IE, n (\%) } \\
\hline Minimal & $9(56.3)$ & $36(67.9)$ & $38(71.7)$ & $0.443^{2}$ & $10(62.5)$ & $35(62.5)$ & $38(76.0)$ & $0.138^{2}$ \\
\hline Moderate & $2(12.5)$ & $10(18.9)$ & $8(15.1)$ & & $1(6.3)$ & $13(23.2)$ & $6(12.0)$ & \\
\hline Extensive & $5(31.3)$ & $7(13.2)$ & $7(13.2)$ & & $5(31.3)$ & $8(14.3)$ & $6(12.0)$ & \\
\hline \multicolumn{9}{|l|}{ PTE, n (\%) } \\
\hline Minimal & $8(50.0)$ & $21(39.6)$ & $35(66.0)$ & $0.075^{2}$ & $8(50.0)$ & $21(37.5)$ & $35(70.0)^{*}$ & $0.014^{2}$ \\
\hline Moderate & $4(25.0)$ & $19(35.8)$ & $8(15.1)$ & & $5(31.3)$ & $20(35.7)$ & $6(12.0)$ & \\
\hline Extensive & $4(25.0)$ & $13(24.5)$ & $10(18.9)$ & & $3(18.8)$ & $15(26.8)$ & $9(18.0)$ & \\
\hline \multicolumn{9}{|l|}{ ITI, n (\%) } \\
\hline Minimal & $7(43.8)$ & $30(56.6)$ & $29(54.7)$ & $0.921^{2}$ & $7(43.8)$ & $30(53.6)$ & $29(58.0)$ & $0.888^{2}$ \\
\hline Moderate & $5(31.3)$ & $14(26.4)$ & $14(26.4)$ & & $5(31.3)$ & $15(26.8)$ & $13(26.0)$ & \\
\hline Extensive & $4(25.0)$ & $9(17)$ & $10(18.9)$ & & $4(25.0)$ & $11(19.6)$ & $8(16.0)$ & \\
\hline \multicolumn{9}{|l|}{ PTI, n (\%) } \\
\hline Minimal & $8(50.0)$ & $24(45.3)$ & $33(62.3)$ & $0.468^{2}$ & $8(50.0)$ & $25(44.6)$ & $32(64.0)$ & $0.304^{2}$ \\
\hline Moderate & $4(25.0)$ & $18(34.0)$ & $12(22.6)$ & & $4(25.0)$ & $20(35.7)$ & $10(20.0)$ & \\
\hline Extensive & $4(25.0)$ & $11(20.8)$ & $8(15.1)$ & & $4(25.0)$ & $11(19.6)$ & $8(16.0)$ & \\
\hline \multicolumn{9}{|l|}{ Desmopl., n (\%) } \\
\hline Minimal & $14(87.5)^{\mathrm{q}}$ & $33(62.3)$ & $15(28.3)$ & $<0.001^{2}$ & $15(93.8)^{\mathrm{q}}$ & $34(60.7)$ & $13(26.0)$ & $<0.001^{2}$ \\
\hline Moderate & $2(12.5)$ & $11(20.8)$ & $21(39.6)$ & & $1(6.3)$ & $14(25.0)$ & $19(38.0)$ & \\
\hline Extensive & $0(0.0)$ & $9(17.0)$ & $17(32.1)$ & & $0(0.0)$ & $8(14.3)$ & $18(36.0)$ & \\
\hline \multicolumn{9}{|l|}{ Neoadj tx., n (\%) } \\
\hline Absent & $13(81.3)$ & $39(73.6)$ & $42(79.2)$ & $0.718^{2}$ & $13(81.3)$ & $43(76.8)$ & $38(76.0)$ & $0.908^{2}$ \\
\hline Present & $3(18.8)$ & $14(26.4)$ & $11(20.8)$ & & $3(18.7)$ & $13(23.2)$ & $12(24.0)$ & \\
\hline \multicolumn{9}{|c|}{$\begin{array}{l}\text { ITB - intratumoral budding; PTB - peritumoral budding; ITL - intratumoral lymphocyte; } P T L-\text { peritumoral lymphocyte; CLI - Crohn-like inflammation; } \\
\text { IE - intratumoral esinophils; PTE - peritumoral eosinophils; ITI - intratumoral overall inflammation; PTI - peritumoral overall inflammation; } \\
\text { Desmopl. - desmoplasia; tx - therapy }\end{array}$} \\
\hline & & & & & & & & \\
\hline
\end{tabular}




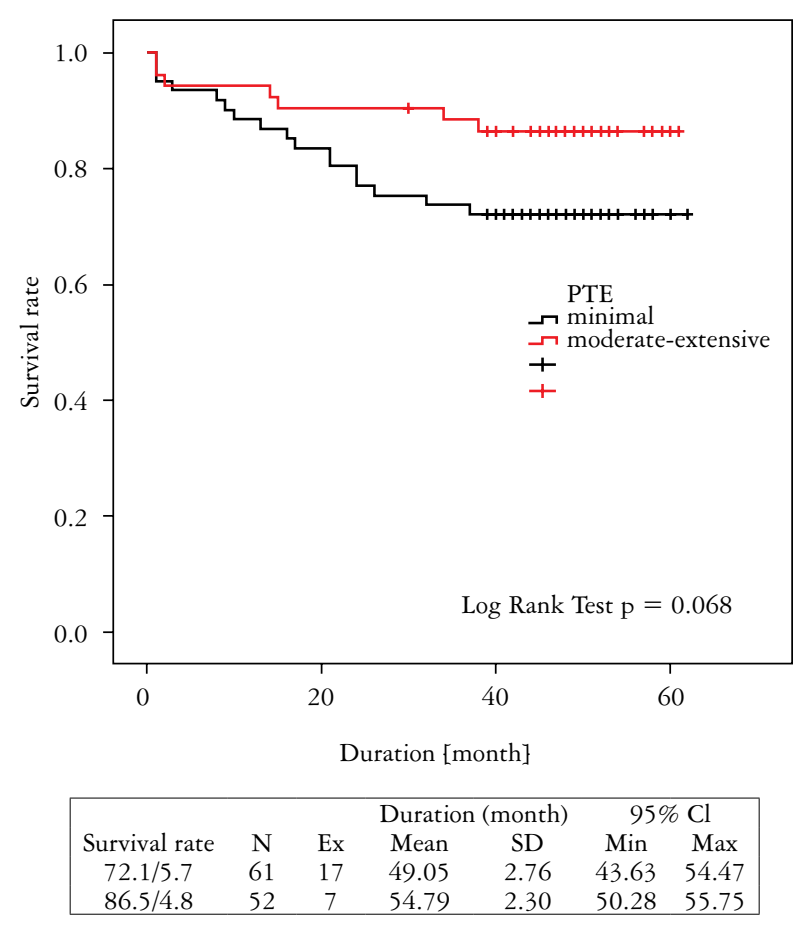

Fig. 7. Survival rate in minimal vs. moderate-extensive peritumoral eosinophils

Minimal PTE was associated with a significantly higher rate of metastasis and pT4 when compared with moderate and extensive PTE $(\mathrm{p}=0.03$ and 0.008 , respectively).

\section{Concordance between ITB and PTB and their association with study parameters}

ITB and PTB were positive totally in $86.9 \%$ of patients with high concordance between ITB and PTB $(92.8 \%, \mathrm{p}=0.000)$. The absence of ITB $(87.5 \%$ vs. $12.5 \%$ and $0.0 \%$, respectively, $\mathrm{p}<0.001$ ) and PTB (93.8\% vs. $6.3 \%$ and $0.0 \%$, respectively, $\mathrm{p}<0.001)$ were both associated with having a higher probability of minimal desmoplasia. The presence of high ITB was associated with a higher rate of minimal PTE $(70.0 \%$ vs. 12.0 and $18.0 \%$, respectively, $\mathrm{p}=0.014)$. No other significant association of PTB or ITB was noted within the study parameters other than those mentioned above (Table III).

\section{Survival data}

Five-year CSS rate was $78.7 \%$, mean (SE) duration of survival was 52.09 (1.87) months. The absence of metastasis when compared to the presence of metastasis was associated with significantly higher 5 -year survival rate $(86.6 \%$ vs. $47.8 \%)$ and a longer duration of survival (mean [SE] 56.0 [1.69] vs. 35.83 [5.06] months) ( $\mathrm{p}<0.001$ ). TNM stage, tumor grade, venous invasion, desmoplasia, neoadjuvant therapy, budding or other tumor-related inflamma- tory parameters had no significant impact on CSS. Albeit not statistically significant, there was a higher rate of 5 -year CSS $(86.5 \%$ vs. $72.15 \%)$ and a longer duration of survival mean [SE] 54.79 [2.30] vs. 49.05 [2.76] months) in the presence of a moderate-extensive rather than a minimal PTE $(\mathrm{p}=0.068)$ (Table IV, Fig. 7).

\section{Cox proportional hazards regression model assessing the prognostic significance of study parameters}

In Cox proportional hazards regression analyses, an increasing age (hazard ratio 1.076, 95\% CI: $1.025-$ $1.130, \mathrm{p}=0.003$ ) and the presence of metastasis (hazard ratio: $4.591,95 \% \mathrm{CI}: 1.814-11.619, \mathrm{p}=0.001)$ were independently associated with poor CSS.

\section{Discussion}

Colorectal cancer is classically seen in aged people and more common in males [1]. In accordance with this, the mean age of our study group was 60 and the majority (59\%) were male.

Our findings revealed that there was an association of an increase in tumor-related inflammatory parameters with lesser likelihood of certain conventional poor prognostic tumor parameters such as $\mathrm{pT} 4$, TNM stage IV, venous invasion and metastasis, and that of higher PTE with lower ITB. This seems in agreement with the consistently reported association of a high-grade peritumoral or intratumoral local inflammatory response with an effective anti-tumor host immune responses and an improved prognosis following a potentially curative resection for CRC $[14,15,16,17]$.

Our findings emphasize the potential role of PTE in prognostic outcome in operated CRC patients given the association of an increase in PTE with a lesser likelihood of ITB and metastasis as well as with a non-significantly for higher 5-year CSS rate and longer duration of CSS.

High IE and PTE counts were shown to be correlated with improved survival in operable CRC in previous studies $[10,14,25,28,29]$, being independent of AJCC/UICC stage [14, 25, 28] and more common for tumors lacking lymph node or distant metastases [30, 31]. However, consistent with our findings non-significantly better survival rather than a significant relationship was also reported for PTE in patients with CRC [18].

In a recent study by Harbaum $e t$ al. [10] on retrospective analysis of peri- and intratumoral eosinophil counts in 381 CRC patients, the presence or an increasing number of eosinophils at the tumor margin was reported to be strongly associated with a favorable tumor phenotype in terms of TNM stage, tumor grade, vascular invasion, and tumor budding as 
Table IV. Survival data in relation to study parameters

\begin{tabular}{|c|c|c|c|c|c|c|c|}
\hline & \multirow[t]{3}{*}{$\mathbf{N}$} & \multirow[t]{3}{*}{ Ex } & \multirow{3}{*}{$\begin{array}{c}\text { 5-YEAR } \\
\text { SURVIVAL RATE } \\
(\% / \mathrm{SE})\end{array}$} & \multicolumn{3}{|c|}{ DURATION OF SURVIVAL (MONTH) } & \multirow[t]{3}{*}{ P VALUE } \\
\hline & & & & \multirow[t]{2}{*}{ Mean (SE) } & \multicolumn{2}{|c|}{$95 \% \mathrm{CI}$} & \\
\hline & & & & & LB & $\mathrm{UB}$ & \\
\hline Overall & 113 & 24 & $78.7 / 3.9$ & $52.09(1.87)$ & 48.43 & 55.75 & - \\
\hline \multicolumn{8}{|l|}{ Grade } \\
\hline Low & 98 & 19 & $80.6 / 4.0$ & $52.53(2.01)$ & 48.60 & 56.47 & 0.270 \\
\hline High & 15 & 5 & $66.7 / 12.0$ & $43.87(4.11)$ & 35.81 & 51.92 & \\
\hline \multicolumn{8}{|l|}{ Gender } \\
\hline Male & 67 & 12 & $82.1 / 4.7$ & $53.36(2.35)$ & 48.75 & 57.97 & 0.312 \\
\hline Female & 46 & 12 & $73.8 / 6.5$ & $49.49(2.97)$ & 43.67 & 55.31 & \\
\hline \multicolumn{8}{|l|}{ Metastasis } \\
\hline Absent & 90 & 12 & $86.6 / 3.6$ & $56.00(1.69)$ & 52.69 & 59.31 & $<0.001$ \\
\hline Present & 23 & 12 & $47.8 / 10.4$ & $35.83(5.06)$ & 25.90 & 45.75 & \\
\hline \multicolumn{8}{|l|}{ Venous invasion } \\
\hline Absent & 71 & 13 & $81.7 / 4.6$ & $52.59(2.20)$ & 48.28 & 56.90 & 0.330 \\
\hline Present & 42 & 11 & $73.7 / 6.8$ & $49.86(3.27)$ & 43.45 & 56.27 & \\
\hline \multicolumn{8}{|l|}{ ITB } \\
\hline Absent-low & 68 & 11 & $83.8 / 4.5$ & $53.12(2.23)$ & 48.74 & 57.50 & 0.123 \\
\hline High & 45 & 13 & $70.9 / 6.8$ & $49.24(3.14)$ & 43.08 & 55.40 & \\
\hline \multicolumn{8}{|l|}{ PTB } \\
\hline Absent-low & 65 & 11 & $83.1 / 4.7$ & $52.65(2.36)$ & 48.02 & 57.27 & 0.223 \\
\hline High & 48 & 13 & $72.7 / 6.5$ & $50.09(2.93)$ & 44.44 & 55.93 & \\
\hline \multicolumn{8}{|l|}{ ITL } \\
\hline Absent & 26 & 5 & $80.8 / 7.7$ & $53.04(3.67)$ & 45.84 & 60.24 & 0.602 \\
\hline Mild-moderate & 62 & 15 & $75.8 / 5.4$ & $49.17(2.73)$ & 43.82 & 54.51 & \\
\hline Extensive & 25 & 4 & $84.0 / 7.3$ & $46.40(2.06)$ & 42.37 & 50.43 & \\
\hline \multicolumn{8}{|l|}{ PTL } \\
\hline Minimal & 59 & 14 & $76.3 / 5.5$ & $51.14(2.60)$ & 46.04 & 56.23 & 0.604 \\
\hline Moderate & 38 & 8 & $78.9 / 6.6$ & $49.92(3.57)$ & 42.93 & 56.91 & \\
\hline Extensive & 16 & 2 & $86.7 / 8.8$ & $57.00(1.98)$ & 53.13 & 60.87 & \\
\hline \multicolumn{8}{|l|}{ CLI } \\
\hline Absent & 40 & 11 & $72.5 / 7.1$ & $46.87(3.70)$ & 39.62 & 54.13 & 0.176 \\
\hline Present & 73 & 13 & $82.1 / 4.5$ & $54.54(1.97)$ & 50.69 & 58.39 & \\
\hline \multicolumn{8}{|l|}{ IE } \\
\hline Minimal & 39 & 17 & $78.5 / 4.6$ & $51.70(2.28)$ & 47.23 & 56.16 & 0.896 \\
\hline Moderate-extensive & 74 & 7 & $79.1 / 7.0$ & $51.36(3.12)$ & 45.26 & 57.47 & \\
\hline \multicolumn{8}{|l|}{ PTE } \\
\hline Minimal & 61 & 17 & $72.1 / 5.7$ & $49.05(2.76)$ & 43.63 & 54.47 & 0.068 \\
\hline Moderate-extensive & 52 & 7 & $86.5 / 4.8$ & $54.79(2.30)$ & 50.28 & 55.75 & \\
\hline \multicolumn{8}{|l|}{ ITI } \\
\hline Minimal & 61 & 14 & $77.0 / 5.4$ & $51.00(2.65)$ & 45.80 & 56.20 & 0.611 \\
\hline Moderate & 30 & 7 & $76.7 / 7.7$ & $50.07(3.76)$ & 42.70 & 57.43 & \\
\hline Extensive & 22 & 3 & $85.9 / 7.5$ & $55.03(2.95)$ & 49.25 & 60.81 & \\
\hline
\end{tabular}


Table IV. Cont.

\begin{tabular}{|c|c|c|c|c|c|c|c|}
\hline & \multirow[t]{3}{*}{$\mathbf{N}$} & \multirow[t]{3}{*}{ Ex } & \multirow{3}{*}{$\begin{array}{c}\text { 5-YEAR } \\
\text { SURVIVAL RATE } \\
(\% / \mathrm{SE})\end{array}$} & \multicolumn{3}{|c|}{ DURATION OF SURVIVAL (MONTH) } & \multirow[t]{3}{*}{$\begin{array}{l}\text { PVALUE } \\
\end{array}$} \\
\hline & & & & \multirow[t]{2}{*}{ MEAn (SE) } & \multicolumn{2}{|c|}{$95 \% \mathrm{CI}$} & \\
\hline & & & & & LB & UB & \\
\hline \multicolumn{8}{|l|}{ PTI } \\
\hline Minimal & 61 & 15 & $75.4 / 5.5$ & $50.15(2.73)$ & 44.80 & 55.49 & 0.624 \\
\hline Moderate & 30 & 5 & $83.3 / 6.8$ & $52.50(3.22)$ & 46.18 & 58.82 & \\
\hline Extensive & 22 & 4 & $81.3 / 8.4$ & $53.74(3.53)$ & 46.82 & 60.67 & \\
\hline \multicolumn{8}{|l|}{ Desmoplasia } \\
\hline Minimal & 57 & 11 & $80.7 / 5.2$ & $52.44(2.45)$ & 47.64 & 57.24 & 0.772 \\
\hline Moderate & 33 & 7 & $78.8 / 7.1$ & $50.36(3.31)$ & 43.88 & 56.84 & \\
\hline Extensive & 23 & 6 & $73.7 / 9.2$ & $49.41(4.59)$ & 40.42 & 58.39 & \\
\hline Neoadj. Tx & & & & & & & 0.818 \\
\hline Absent & 88 & & $78.4 / 4.4$ & $51.71(2.17)$ & 47.45 & 55.98 & \\
\hline Present & 25 & & $80.0 / 8.0$ & $48.60(3.08)$ & 42.55 & 54.65 & \\
\hline
\end{tabular}

well as with improved survival [10]. The authors also noted that although the PTE count correlated with the intensity of the overall inflammatory cell reaction, it was independently associated with the outcome [10]. In our study, although we found that high PTE was strongly associated with better survival rates, probably due to the relatively small sample size, we did not obtain statistically significant $p$ values $(\mathrm{p}=0.068)$. Similarly, in our cohort, although extensive CLI was also associated with a decreased rate of metastasis and TNM stage 4, only PTE was associated significantly with ITB and potential survival. This seems to support the potential role of eosinophil infiltration as an antitumoral mechanism independent of overall host inflammatory cell reaction in CRCs [10, 32].

In a recent study by Briede et al. on retrospective analysis of 553 CRC cases, high grade peritumoral inflammation was associated with beneficial morphologic CRC features, including less frequent manifestations of invasion [19]. In our study, we found no association of ITI and PTI with any of the tumor prognostic parameters. This may be due to high density of neutrophil leukocytes in ITI and PTI, since there are controversial findings on the prognostic value of neutrophils in CRC [33, 34]. These controversies may be explained by the duality of neutrophils comprising both a tumor suppressive $\mathrm{N} 1$ population and tumor supportive N2 neutrophils [35].

Nonetheless, while the evaluation of PTE seems to be a promising tool with potential to improve risk stratification in CRC patients [10], inconsistency regarding its relationship on overall inflammation or staging of the disease $[10,17,28,29]$ is considered to challenge its incorporation in the routine prognostic assessment in clinical practice [17].

In a study by Nagtegaal et al. [28] in 160 CRC patients, an increasing peritumoral, but not intratumoral eosinophil count was reported to be associated with better CSS and lower rates of recurrence. This effect was shown to be dependent on both TNM stage and overall inflammatory cell reaction [28]. In another study carried out by Fisher et al. [29] higher numbers of eosinophils were found to be associated with better overall survival, when dependent at the tumor stage but not on the overall inflammatory cell reaction [29].

In a meta-analysis of 30 studies on 2988 patients on the impact of tumor-infiltrating inflammation on survival outcomes in terms of generalized tumor inflammatory infiltrate $(\mathrm{n}=12)$ and T lymphocyte subsets $(\mathrm{n}=18)$, the authors concluded the association of high density of generalized tumor inflammatory infiltrate to be a good prognostic marker for CRC. This was due to its significant association with improved survival, and emphasized a need for further prospective studies on subsets of $\mathrm{T}$ lymphocytes due to significant heterogeneity and an insufficient number of studies [36].

In addition, mechanisms of antitumoral activity exhibited by eosinophils also remain not elucidated, while emerging evidence indicates that they may exert their anti-tumor effect not only through their cytotoxicity (such as TNF- $\alpha$, granzyme, cationic proteins and IL-8), but also via immunomodulatory mechanisms including secretion of T-cell cytokines, activation of dendritic cells or through antigen presentation to T-cells $[10,17,37,38]$. Additionally, a plethora of factors produced by cancer and immune 
cells can attract and/or activate eosinophils in the tumor microenvironment [38].

An association of high-grade tumor infiltrating lymphocytes with a higher likelihood of deriving a survival benefit from adjuvant chemotherapy in patients undergoing curative resection of CRC was reported $[17,39]$. Therefore, the increase in ITL with higher tumor grade and lower likelihood of venous invasion in our cohort seems notable. This seems to also emphasize the likelihood of the presence of a distinct lymphocytic infiltrative pattern rather than specific tumor infiltrating lymphocytes to be the most important determinant of survival $[14,40]$.

In a retrospective analysis of 120 patients with AJCC/UICC stage II colorectal cancer by Betge et al., high score tumor budding was reported to be significantly associated with tumor grade and lymphovascular invasion and thought to be an independent predictor of disease progression and cancer-related death [41]. Our findings revealed a high concordance between ITB and PTB, in parallel to their similar pathogenesis as morphologic manifestations of epithelial-mesenchymal transition [42]. Both high ITB and high PTB were associated with higher rates of pT4 stage, venous invasion and moderate-to-extensive desmoplasia. This indicates that tumor budding is frequently found in tumors with an invasive growth pattern and desmoplastic stromal response. Neither ITB nor PTB was associated with a metastasis rate in our cohort despite the reported association of tumor budding with an increased probability for metastatic dissemination to lymph nodes and distant organs. Our findings were thus compatible with the literature [43].

The presence of higher ITB in patients with minimal as compared to moderate-to-extensive PTE in our cohort seems to support the potential role of eosinophil infiltration as an antitumoral mechanism [10]. It also seems notable given the infrequently encountered tumor budding in patients with a strong peritumoral infiltration caused by $\mathrm{CD} 8+$ cytotoxic $\mathrm{T}$ cells, indicating the likelihood of an interaction between tumor budding cells and the host immune system as two opposing sides of an attacker-defender model [13, 43].

Our findings emphasized the potential role of additional prognostic parameters related to tumor microenvironment alongside the conventional morphological prognostic parameters in the identification of high-risk pathological features and in guiding provision of adjuvant therapy in CRCs $[10,14,15]$.

The retrospective and single center nature of our study was an important limitation and might have influenced our findings in establishing the temporality between cause and effect as well as generalizing our findings to an overall CRC population. The other limitation of this study was the relatively small sample size that might have prevented from obtaining statistical significance concerning prognostic role of tumor inflammatory parameters. The inclusion of patients with and without neoadjuvant therapy together was another limitation of the study. Nevertheless, despite these limitations, given the paucity of the solid information available in this area, our findings represent a valuable contribution to the literature by providing data on assessment of the prognostic role of tumor-associated inflammatory infiltrates based on standardized criteria and being stratified by location (intratumoral, peritumoral) and inflammatory cell subset of both the adaptive and innate immune system (lymphocytes, eosinophils).

In conclusion, our findings revealed that low score tumoral budding and an increase in tumor-related inflammatory parameters were associated with a lesser likelihood of conventional poor prognostic tumor parameters such as pT4, TNM stage IV, venous invasion and metastasis. Nonetheless, given the association of an increase in peritumoral eosinophil infiltrates with a lower probability of intratumoral budding, pT4, metastasis and for better survival rates, our findings emphasize the potential role of peritumoral eosinophils independent of the overall inflammatory reaction. This can be considered as an additional prognostic parameter related to the tumor microenvironment in better stratification of progression and guiding provision of adjuvant therapy tailored to individualized risk.

\section{The authors declare no conflict of interest.}

\section{References}

1. Rawla P, Sunkara T, Barsouk A. Epidemiology of colorectal cancer: incidence, mortality, survival and risk factors. Gastroenterology Rev 2019; 14: 89-103.

2. Lee $\mathrm{CH}$, Tseng PL, Tung HY, et al. Comparison of risk factors between colon cancer and rectum cancer in a single medical center hospital, Taiwan. Arch Med Sci 2020; 16: 102-111.

3. Malyarchuk BA, Grzybowski T, Derenko MV, et al. Mitochondrial DNA variability in Poles and Russians. Ann Hum Genet 2002; 66: 261-283

4. Mohammed F, Rezaee Khorasany AR, Mosaileby E, et al. Mitochondrial A12308G alteration in tRNA(Leu (CUN)) in colorectal cancer samples. Diagn Pathol 2015; 10: 115.

5. Kumar B, Bhat ZI, Bansal S, et al. Asscociation of mitochondrial copy number variation and T16189C polymorphism with colorectal cancer in North Indian population. Tumour Biol 2017; 39: 1010428317740296.

6. Webb E, Broderick P, Chandler I, et al. Comprehensive analysis of common mitochondrial DNA variants and colorectal cancer risk. Br J Cancer 2008; 99: 2088-2093.

7. Skonieczna K, Jawien A, Marszalek A, et al. Mitogenome germline mutations and colorectal cancer risk in Polish population. Arch Med Sci 2020; 16: 366-373.

8. Compton CC. Optimal pathologic staging: defining stage II disease. Clin Cancer Res 2007; 13: 6862-6870.

9. Barresi V, Reggiani Bonetti L, Ieni A, et al. Poorly differentiated clusters: clinical impact in colorectal cancer. Clin Colorectal Cancer 2017; 16: 9-15. 
10. Harbaum L, Pollheimer MJ, Kornprat P, et al. Peritumoral eosinophils predict recurrence in colorectal cancer. Mod Pathol 2015; 28: 403-413.

11. O'Connell JB, Maggard MA, Ko JY. Colon cancer survival rates with the new American Joint Committee on Cancer sixth edition staging. J Natl Cancer Inst 2004; 96: 1420-1425.

12. Wang LM, Sheahan K. Prognostic markers in colorectal pathology: is morphology enough? Diag Pathol 2011; 17: 386-394.

13. Lugli A, Karamitopoulou E, Panayiotides I, et al. CD8+ lymphocytes/tumour-budding index: an independent prognostic factor representing a 'pro-/anti-tumour' approach to tumour host interaction in colorectal cancer. Br J Cancer 2009; 101: 1382-1392.

14. Roxburgh CS, McMillan DC. The role of the in situ local inflammatory response in predicting recurrence and survival in patients with primary operable colorectal cancer. Cancer Treat Rev 2012; 38: 451-466.

15. Galon J, Costes A, Sanchez-Cabo F, et al. Type, density and location of immune cells within human colorectal tumors predict clinical outcome. Science 2006; 313: 1960-1964.

16. Pagès $\mathrm{F}$, Mlenic $\mathrm{B}$, Marliot $\mathrm{F}$, et al. International validation of the consensus Immunoscore for the classification of colon cancer: a prognostic and accuracy study. Lancet 2018; 391: 2128- 2139 .

17. Prizment AE, Vierkant RA, Smyrk TC, et al. Tumor eosinophil infiltration and improved survival of colorectal cancer patients: Iowa Women's Health Study. Mod Pathol 2016; 29: 516-527.

18. Klintrup K, Makinen JM, Kauppila S, et al. Inflammation and prognosis in colorectal cancer. Eur J Cancer 2005; 41: 2645 2654.

19. Briede I, Strumfa I, Vanags A, et al. The association between inflammation, epithelial mesenchymal transition and stemness in colorectal carcinoma. J Inflamm Res 2020; 13: 15-34.

20. Amin MB, Edge SB, Greene FL, et al. AJCC Cancer Staging Manual, $8^{\text {th }}$ ed. Springer, New York 2017.

21. Hamilton SR, Bosman FT, Boffetta P. Carcinoma of the colon and rectum. Bosman FT, Carneiro F, Hruban RH (eds.). World Health Organization classification of tumours of the digestive system. IARC, Lyon 2010; 131-181.

22. Dawson H, Kirsch R, Driman DK, et al. Optimizing the detection of venous invasion in colorectal cancer: the Ontario, Canada, experience and beyond. Front Oncol 2015; 4: 354.

23. Kirch R, Messenger D, Riddell R, et al. Venous invasion in colorectal cancer: Impact of an elastin stain on detection and interobserver agreement among gastrointestinal and nongastrointestinal pathologists. Am J Surg Pathol 2013; 37: 200-210.

24. Graham RP, Vierkant RA, Tillmans LS, et al. Tumor budding in colorectal carcinoma: confirmation of prognostic significance and histologic cutoff in a population-based cohort. Am J Surg Pathol 2015; 39: 1340-1346.

25. Fernández-Aceñero MJ, Galindo-Gallego M, Sanz J, Aljama A. Prognostic influence of tumor-associated eosinophilic infiltrate in colorectal carcinoma. Cancer 2000; 88: 1544-1548.

26. College of American Pathologists protocol for the examination of specimens from patients with primary carcinoma of the colon and rectum. Based on AJCC/UICC TNM, 8th edition Protocol web posting date: February 2020 Available from: https:// documents.cap.org/protocols/cp-gilower-colonrectum-biopsy-20-4100.pdf.

27. Huh JW, Lee JH, Kim HR. Prognostic significance of tumor-infiltrating lymphocytes for patients with colorectal cancer. Arch Surg 2012; 147: 366-371.

28. Nagtegaal ID, Marijnen CA, Kranenbarg EK, et al. Local and distant recurrences in rectal cancer patients are predicted by the nonspecific immune response; specific immune response has only a systemic effect - a histopathological and immunohistochemical study. BMC Cancer 2001; 1: 7.
29. Fisher ER, Paik SM, Rockette H, et al. Prognostic significance of eosinophils and mast cells in rectal cancer: findings from the National Surgical Adjuvant Breast and Bowel Project (protocol R-01). Hum Pathol 1989; 20: 159-163.

30. McGinnis MC Jr., Bradley EL, Pretlow TP, et al. Correlation of stromal cells by morphometric analysis with metastatic behavior of human colonic carcinoma. Cancer Res 1989; 49: 5989-5993

31. Pretlow TP, Boohaker EA, Pitts AM, et al. Heterogeneity and subcompartmentalization in the distribution of eosinophils in human colonic carcinomas. Am J Pathol 1984; 116: 207-213.

32. Richards $\mathrm{CH}$, Flegg KM, Roxburgh CS, et al. The relationships between cellular components of the peritumoural inflammatory response, clinicopathological characteristics and survival in patients with primary operable colorectal cancer. Br J Cancer 2012; 106: 2010-2015.

33. Rao H, Chen J, Li M, et al. Increased intratumoral neutrophil in colorectal carcinomas correlates closely with malignant phenotype and predicts patients' adverse prognosis. PLoS One 2012; 7: e30806.

34. Akishima-Fukasawa Y, Ishikawa Y, Akasaka Y, et al. Histopathological predictors of regional lymph node metastasis at the invasive front in early colorectal cancer. Histopathology 2011; 59: 470-481.

35. Mizuno R, Kawada K, Itatani Y, et al. The role of tumor-asscociated neutrophils in colorectal cancer. Int J Mol Sci 2019; 20: 529.

36. Mei Z, Liu Y, Liu C, et al. Tumour-infiltrating inflammation and prognosis in colorectal cancer: systematic review and meta-analysis. Br J Cancer 2014; 110: 1595-1605.

37. Kita H. Eosinophils: Multifaceted biological properties and roles in health and disease. Immunol Rev 2011; 242: 161-177.

38. Varricchi G, Galdiero MR, Loffredo S, et al. Eosinophils:the unsung heroes in cancer? Oncoimmunology 2018; 7: e139134.

39. Morris M, Platell C, Iacopetta B. Tumor-infiltrating lymphocytes and perforation in colon cancer predict positive response to 5-fluorouracil chemotherapy. Clin Cancer Res 2008; 14: 1413-1417.

40. Ogino S, Nosho K, Irahara N, et al. Lymphocytic reaction to colorectal cancer is associated with longer survival, independent of lymph node count, microsatellite instability, and $\mathrm{CPG}$ island methylator phenotype. Clin Cancer Res 2009; 15: 64126420.

41. Betge J, Kornprat P, Pollheimer MJ, et al. Tumor budding is an independent predictor of outcome in AJCC/UICC stage II colorectal cancer. Ann Surg Oncol 2012; 19: 3706-3712.

42. Lugli A, Kirsch R, Ajioka Y, et al. Recommendations for reporting tumor budding in colorectal cancer based on the International Tumor Budding Consesnsus Conference (ITBCC) 2016. Mod Pathol 2017; 30: 1299-1311.

43. Koelzer VH, Zlobec I, Lugli A. Tumor budding in colorectal cancer - ready for diagnostic practice? Hum Pathol 2016; 47: 4-19.

\section{Address for correspondence}

\section{Saime Ramadan}

Department of Pathology,

Baskent University Istanbul Hospital

Altunizade Mah. 7. Kisikli cad. Oymac1 Sk. 34662

Uskudar/Istanbul, Turkey

tel. +905335255563

fax +902164743149

e-mail: drsaimeramadan@yahoo.com 